\title{
Index Structures for Data Warehouses
}

\author{
Dissertation \\ am \\ Fachbereich für Mathematik und Informatik \\ an der \\ Freien Universität Berlin
}

eingereicht am 22.12.1999

von

Marcus Jürgens

\section{Betreuer}

Prof. Dr. Hans-Joachim Lenz (Freie Universität Berlin)

Prof. Dr. Heinz Schweppe (Freie Universität Berlin)

Prof. Dr. Johann Christoph Freytag (Humboldt-Universität zu Berlin) 
Tag der mündlichen Prüfung: 16. Feb. 2000 
to Chris 


\section{Abstract}

This thesis investigates which index structures support query processing in typical data warehouse environments most efficiently. Data warehouse applications differ significantly from traditional transaction-oriented operational applications. Therefore, the techniques applied in transaction-oriented systems cannot be used in the context of data warehouses and new techniques must be developed.

The thesis shows that the time complexity for the computation of optimal tree-based index structures prohibits its use in real world applications. Therefore, we improve heuristic techniques (e.g. $R^{*}$-tree) to process range queries on aggregated data more efficiently. Experiments show the benefits of this approach for different kinds of typical data warehouse queries. Performance models estimate the behavior of standard index structures and the behavior of the extended index structures. We introduce a new model that considers the distribution of data. We show experimentally that the new model is more precise than other models known from literature. Two techniques compare two tree-based index structures with two bitmap indexing techniques. The performance of these index structures depends on a set of different parameters. Our results show which index structure performs most efficiently depending on the parameters. 


\section{Acknowledgements}

I am very grateful to have had the opportunity to write my $\mathrm{Ph}$. D. Thesis under the supervision of Professor Hans-Joachim Lenz who brought the area of data warehouses to my attention. In countless meetings he gave me helpful feedback. I would like to thank Professor Heinz Schweppe for his constructive suggestions and the invitation to cooperate with the database group at the Freie Universität Berlin. Professor Freytag supported me with beneficial ideas and outstanding comments.

The graduate school in Distributed Information Systems would not be possible in this efficient form without its speaker Professor Oliver Günther. His commitment made this school a constructive and pleasant environment.

I would like to express my thanks to all members of database groups participating in this graduate school for their interesting and encouraging talks and discussions. In particular, I am grateful for the constructive discussions with Agnès Voisard and Annika Hinze. The Deutsche Forschungsgemeinschaft (DFG) supported me as a fellowship recipient. Professor Joseph Bronstad and Leslie Hazelwood did not resign trying to correct my English. 


\section{Contents}

1. Introduction 1

1.1. Goals . . ...................... 2

1.2. Outline ...................... 3

2. State of the Art of Data Warehouse Research 5

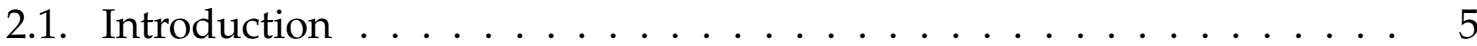

2.2. Traditional transaction-oriented systems . . . . . . . . . . . . . 5

2.3. Data warehouses for decision support . . . . . . . . . . . . . 7

2.4. OLAP vs. OLTP . . . . . . . . . . . . . . . . . . . . . . . 9

2.5. Accelerating query speed $\ldots \ldots \ldots \ldots \ldots$

2.5.1. Denormalized schemas . . . . . . . . . . . . . . . . . 10

2.5.2. Materialized views . . . . . . . . . . . . . . . . . . . . 11

2.5.3. No locking . . . . . . . . . . . . . . . . . . 13

2.5.4. On-line aggregation . . . . . . . . . . . . . . 13

2.5.5. Index structures . . . . . . . . . . . . . . . . . . . . 13

2.6. Summary . . . . . . . . . . . . . . . . . . . . . . . . . 14

3. Data Storage and Index Structures 15

3.1. Introduction . . . . . . . . . . . . . . . . . . 15

3.2. Memory hierarchy . . . . . . . . . . . . . . . . . . . 15

3.3. Mechanics of disks . . . . . . . . . . . . . . . . . . 16

3.4. Data space and queries . . . . . . . . . . . . . . . . . . . 18

3.4.1. Data space . . . . . . . . . . . . . . . . . 18

3.4.2. Queries . . . . . . . . . . . . . . . . . . . 18

3.5. Tree-based indexing . . . . . . . . . . . . . . . . . . . . . . . . . 19

3.5.1. Top-down, bottom-up, and bulk loading . . . . . . . . . . 20

3.5.2. Point quadtrees . . . . . . . . . . . . . . . . 21

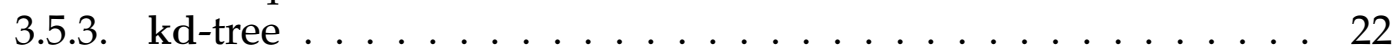

3.5.4. kdb-tree . . . . . . . . . . . . . . . . 22

3.5.5. R-tree ...................... 22

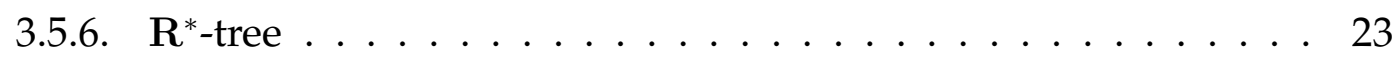

3.5.7. Other relatives of the $\mathbf{R}$-tree family and other tree structures . 24

3.5.8. Generic tree structures . . . . . . . . . . . . . . . . . 26

3.6. Bitmap indexing . . . . . . . . . . . . . . 27 
3.6.1. Standard bitmap indexing . . . . . . . . . . . . . . . 27

3.6.2. Multi-component equality encoded bitmap index . . . . . . . . 29

3.6.3. Range-based encoding . . . . . . . . . . . . . . . . . . 31

3.6.4. Multi-component range-based encoding . . . . . . . . . 32

3.6.5. Other compression techniques / combination of bitmaps and

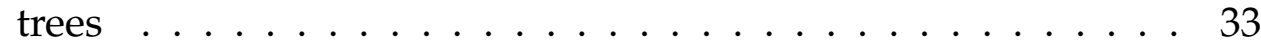

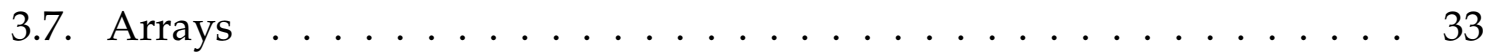

3.8. Summary . . . . . . . . . . . . . . . . . . 34

4. Mixed Integer Problems for Finding Optimal Tree-Based Index Structures 35

4.1. Introduction . . . . . . . . . . . . . . . . . 35

4.2. Optimization problem parameters . . . . . . . . . . 35

4.3. Mapping into a mixed integer problem . . . . . . . . . . . . . . 36

4.4. Problem complexity . . . . . . . . . . . . . . . . . . 38

4.5. Model evaluation . . . . . . . . . . . . . . . . . . . . . . 39

4.6. Summary . . . . . . . . . . . . . . . . . . . . 41

5. Aggregated Data in Tree-Based Index Structures 43

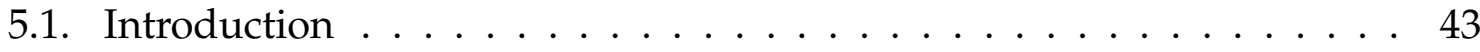

5.2. "Fit for aggregation" access method . . . . . . . . . . . . . . 47

5.3. Materialization of data . . . . . . . . . . . . . . . . . . . 48

5.4. Modified operations . . . . . . . . . . . . . . . . . 50

5.4.1. Insert operation . . . . . . . . . . . . . . . 50

5.4.2. Delete operation . . . . . . . . . . . . . . . 51

5.4.3. Update operation . . . . . . . . . . . . . . . . . . . 51

5.4.4. Creating index structures, bottom-up index structures . . . . 51

5.4.5. Point query algorithm . . . . . . . . . . . . . . . . 52

5.4.6. Range query algorithm . . . . . . . . . . . . . . . 52

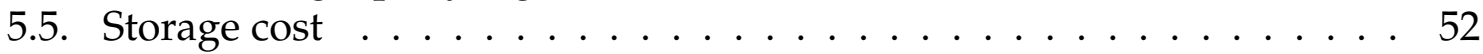

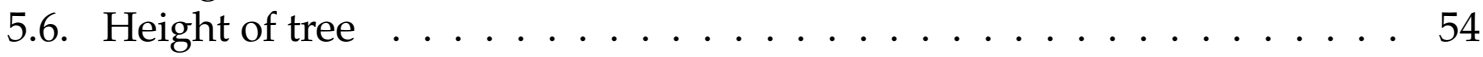

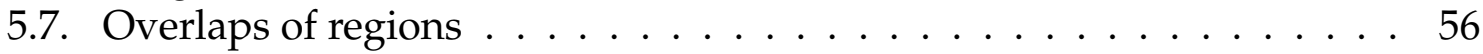

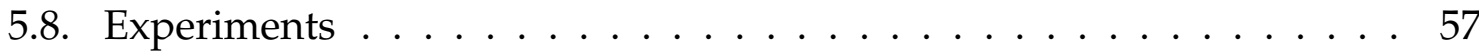

5.8.1. Cost model . . . . . . . . . . . . . . . . . . 57

5.8.2. Physical index structure . . . . . . . . . . . . . . 58

5.8.3. Implementation . . . . . . . . . . . . . . . . 58

5.8.4. Generation of test data . . . . . . . . . . . . . . . 58

5.8.5. Query profile . . . . . . . . . . . . . . . . . 60

5.8.6. Results of experiments . . . . . . . . . . . . . . . 60

5.9. Summary . . . . . . . . . . . . . . . . . . . 62

6. Performance Models for Tree-Based Index Structures 63

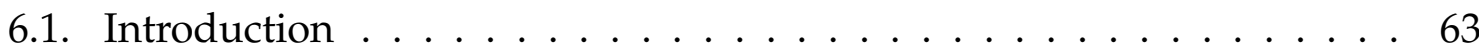

6.2. Fit for modeling . . . . . . . . . . . . . . . . 63 
6.3. Performance models for access leaf nodes . . . . . . . . . . . . . . . . 64

6.3.1. GRID model . . . . . . . . . . . . . . . . . . . . . . . . 64

6.3.2. SUM model . . . . . . . . . . . . . . . . . . . . . . 66

6.3.3. Equivalence of GRID model and SUM model . . . . . . . . . 67

6.3.4. FRACTAL model . . . . . . . . . . . . . . . . . . . . . . . . 69

6.3.5. Equivalence between FRACTAL model, SUM model, and

GRID model . . . . . . . . . . . . . . . . . . . . . . . 71

6.4. PISA model . . . . . . . . . . . . . . . . . . . . 71

6.5. Computational Efficiency of SUM model and PISA model . . . . . . . 74

6.6. Adapting PISA model to different distributions . . . . . . . . . . . . . 76

6.6.1. Uniformly distributed data . . . . . . . . . . . . 76

6.6.2. Skewed data . . . . . . . . . . . . . . . . . . 77

6.6.3. Normally distributed data . . . . . . . . . . . . . . . . . 79

6.7. Model evaluation . . . . . . . . . . . . . . . . . . . 80

6.7.1. Uniformly distributed data . . . . . . . . . . . . 8 81

6.7.2. Skewed data . . . . . . . . . . . . . . . . . . . 81

6.7.3. Normally distributed data . . . . . . . . . . . . . . . . 84

6.8. PISA model for dependent data . . . . . . . . . . . . . . . . 84

6.9. Extension of models . . . . . . . . . . . . . . . . . . . 85

6.10. Applications of models . . . . . . . . . . . . . . . . 86

6.10.1. Savings of $\mathbf{R}^{*}{ }$-tree depending on the query box size and form 86

6.10.2. Savings of $\mathbf{R}_{a}^{*}$-tree depending on the number of dimensions . 86

6.11. Summary . . . . . . . . . . . . . . . . . . 87

7. Techniques for Comparing Index Structures 89

7.1. Introduction . . . . . . . . . . . . . . . . . . . . . . . . 89

7.2. Experimental parameters . . . . . . . . . . . . . . . . . . . 89

7.2.1. Data specific parameters . . . . . . . . . . . . . . . . . . . 89

7.2.2. Query specific parameters . . . . . . . . . . . . . . . . . 90

7.2.3. System specific parameters . . . . . . . . . . . . . . . . 91

7.2.4. Disk specific parameters . . . . . . . . . . . . . . . . 91

7.2.5. Configuration . . . . . . . . . . . . . . . 92

7.3. Index structures and time estimators . . . . . . . . . . . . 93

7.3.1. Time Measures for tree-based index structures . . . . . . . . . 93

7.3.2. Time measures for bitmap indexing techniques . . . . . . . . . 94

7.4. Classification trees . . . . . . . . . . . . . . . . 96

7.4.1. Applied methods . . . . . . . . . . . . . . . . . 9 97

7.4.2. Value sets of Parameters . . . . . . . . . . . . . 97

7.4.3. Results . . . . . . . . . . . . . . . . . . . . 99

7.5. Statistics in two dimensions . . . . . . . . . . . . . 102

7.5.1. Sum aggregation . . . . . . . . . . . . . 103

7.5.2. Median aggregation . . . . . . . . . . . . . 103

7.5.3. Count aggregation . . . . . . . . . . . . . . 103

7.5.4. Results . . . . . . . . . . . . . . . . 106 


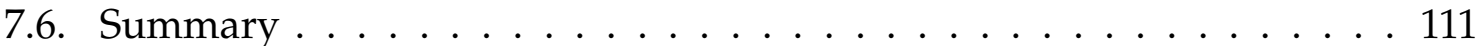

$\begin{array}{ll}\text { 8. Conclusion and Outlook } & 113\end{array}$

$\begin{array}{ll}\text { Bibliography } & 116\end{array}$

$\begin{array}{ll}\text { Index } & 125\end{array}$

$\begin{array}{ll}\text { A. List of Symbols } & 127\end{array}$

B. Approximation of PISA Model 133

\section{Ph D Related Material}

Zusammenfassung der Ergebnisse . . . . . . . . . . . . . . 135

Lebenslauf . . . . . . . . . . . . . . . . . . 136

Verwendete Hilfsmittel . . . . . . . . . . . . . . . . . . . . 137 